\title{
Evaluación del rendimiento energético del bagazo de caña de un ingenio azucarero vs su aprovechamiento mediante gasificación
}

\section{Evaluation of the energy performance of sugarcane bagasse from a sugar mill vs its use through gasification}

\author{
Iván Solano ${ }^{1}$, Orlando Aguilar ${ }^{2}$, Camila Domínguez ${ }^{2}$, Gabriela Ramírez ${ }^{2}$, Orlando Aguilar $^{3 *}$ \\ ${ }^{1}$ Licenciatura en Ingeniería Electromecánica, Universidad Tecnológica de Panamá; ${ }^{2}$ Licenciatura en Ingeniería Mecánica, \\ Universidad Tecnológica de Panamá; ${ }^{3}$ Facultad de Ingeniería Mecánica y director del Grupo de Investigación en Energía \\ Renovable y Ambiente (GIERA), Universidad Tecnológica de Panamá.
}

\begin{abstract}
Resumen El hombre ha desarrollado una dependencia hacia los combustibles fósiles desde la revolución industrial (siglo XVIII); de este hecho surge una necesidad actual cuya tendencia es desarrollar sistemas de generación de energía con fundamento en la utilización de energías renovables. La biomasa es un recurso que presenta una gran disponibilidad alrededor de todo el mundo, es por esto que se ha tenido el objetivo de implementar tecnologías o procesos, de tal manera que se pueda aprovechar este recurso tan abundante. Es así como la gasificación se ha ido convirtiendo en un proceso líder en este aspecto. Un tipo de biomasa común en Panamá es el bagazo de caña, que no es más que el residuo que queda como producto una vez se ha extraído el jugo de la caña de azúcar. El objetivo de esta investigación es hacer una evaluación del rendimiento energético del bagazo de caña utilizado en los ingenios azucareros de Panamá, tal y como se usa actualmente para la combustión y generación de energía, de tal modo que podamos compararlos con el rendimiento energético que presentaría cuando el bagazo de caña sea gasificado, para determinar cuál proceso es el más eficiente para la producción de energía eléctrica. Para llevar a cabo esta idea se planea tomar como muestra por lo menos dos ingenios azucareros en nuestro país, ya que las propiedades del bagazo de caña podrían variar de un ingenio a otro, según la variedad de la caña. Una vez hecha la comparación de rendimiento energético se podrá recomendar a los ingenios los cambios de tecnología pertinentes para mejorar su operación.
\end{abstract}

Palabras clave Biomasa, gasificación, bagazo de caña, ingenio, poder calorífico, syngas.

\begin{abstract}
Humans have developed a dependency on fossil fuels since the industrial revolution (18th century); from this fact a current need arises whose tendency is to develop energy generation systems based on the use of renewable energy. Biomass is a resource that is widely available around the world, which is why the objective has been to implement technologies or processes, so that this abundant resource can be used. This is how gasification has become a leading process in this regard. A common type of biomass in Panama is sugarcane bagasse, which is nothing more than the residue that remains as a product once the sugar cane juice has been extracted. The objective of this research is to make an evaluation of the energy performance of the sugarcane bagasse used in the sugar mills of Panama, as it is currently used for combustion and energy generation, so that we can compare them with the energy performance that it would present when the sugarcane bagasse is gasified, to determine which process is the most efficient for the production of electrical energy. To carry out this idea, it is planned to take as a sample at least two sugar mills in our country, since the properties of cane bagasse could vary from one mill to another, depending on the variety of the cane. Once the energy performance comparison has been made, the mills can be recommended for the relevant technology changes to improve their operation.
\end{abstract}

Keywords Biomass, gasification, sugar cane bagasse, sugar cane mill, calorific value, syngas.

*Corresponding author: orlando.aguilar@utp.ac.pa

\section{Introducción}

La utilización de energía no renovable es algo que se ha venido dando desde hace dos siglos, pero hemos tardado demasiado tiempo en darnos cuenta lo perjudicial que es este tipo de energía para el ser humano y el medio ambiente. En los años recientes, el mundo ha estado buscando nuevas fuentes de energía que, aparte de ser renovables, no sean dañinas al medio ambiente. Uno de los recursos de más interés en los recientes años es la biomasa. La biomasa es un recurso renovable que proviene de las plantas verdes y se produce de la conversión de energía solar en materia orgánica mediante el proceso de fotosíntesis [1]. Este tipo de recurso renovable en 
específico presenta una gran disponibilidad alrededor de todo el mundo y además posee una capacidad para producir energía eléctrica debido a su poder calorífico, lo cual la hace más eficiente que los combustibles fósiles convencionales [2]. Un proceso con el que se puede aprovechar ese poder calorífico tan alto de la biomasa es la gasificación, que consiste en un proceso térmico en el cual el combustible sólido (biomasa) mediante el uso de un gasificador se convierte en un gas llamado syngas que puede ser usado para producción de potencia y calor [2] [3]. El syngas, como se mencionó anteriormente, es el producto de la gasificación y no es más que un tipo de gas constituido por gases que son tanto combustibles como no combustibles, pero los compuestos más comunes que se encuentran en dicho gas son: hidrógeno, monóxido de carbono y dióxido de carbono [4]. Los ingenios azucareros son un tipo de planta que se encarga del procesamiento de la caña de azúcar para producir distintos productos. Una vez dicha caña de azúcar se ha procesado, el residuo que queda como resultado del proceso es lo que se conoce como bagazo de caña de azúcar. El propósito de esta investigación es hacer un estudio de la eficiencia energética del bagazo de caña usado como tal para la producción de energía eléctrica en los ingenios de Panamá y compararlo con la eficiencia que podría brindar el mismo bagazo de caña al ser gasificado en su estado común de fibra y peletizado.

\section{Antecedentes}

La gasificación de biomasa es un proceso del cual se han comenzado a hacer muchas investigaciones desde hace aproximadamente cinco décadas. No obstante, es un proceso cuyo origen se da hace alrededor de 180 años atrás. El primer gasificador construido fue elaborado por una empresa alemana llamada Bischof en el año 1839 y era un gasificador de carbón [3]. La siguiente compañía en desarrollar un gasificador, también alemana, fue Siemens a mediados del siglo XIX y su principal uso era la producción de syngas que era el combustible de hornos en distintas industrias. La necesidad por producir un syngas que fuese menos perjudicial para el ambiente comenzó a ser trabajada a finales del siglo XIX cuando una empresa inglesa desarrolla un sistema para que se produzca un gas más limpio, lo que permitió que se pudiera usar el syngas como combustible para motores de combustión interna [5]. La gasificación se convirtió en un proceso líder debido a que se gasificaba carbón vegetal para la producción de combustible que sería utilizado en distintos vehículos tanto terrestres como acuáticos y esto se debe a que fue una época donde no había mucho petróleo. Sin embargo, cuando la segunda guerra mundial terminó, los combustibles fósiles comenzaron a ser usados nuevamente y se abandonó de cierto modo la gasificación de biomasa [2]. Recientemente, solo se había usado, a mediados del siglo pasado, para la obtención de hidrógeno, a partir del carbón, para la confección de abonos. No fue hasta hace unos 20 años que se comenzó a utilizar el proceso de gasificación para la producción de energía eléctrica, lo que dio como resultado a que actualmente, se cuente con una planta de biomasa de pellets de madera en el Reino Unido con una capacidad de 740 MW [1].

Los estudios en la actualidad van dirigidos a distintas áreas para el aprovechamiento de la biomasa. Entre esos estudios, los más relevantes son la producción de energía usando biomasa peletizada, el tratamiento del syngas durante el proceso de gasificación para mejorar sus propiedades y el diseño de gasificadores para aumentar su eficiencia.

\section{Marco Teórico}

\subsection{Biomasa}

La biomasa es un término que se usa para describir todo material orgánico que proviene de seres vivos. La biomasa vegetal se produce cuando las plantas convierten la energía solar mediante el proceso de la fotosíntesis en materia orgánica. Se puede considerar como materia orgánica, en la cual la energía solar está almacenada en enlaces químicos, y cuando estos enlaces se rompen, la energía almacenada es liberada [5]. Además de la disponibilidad a nivel mundial de la biomasa, también presenta otra ventaja sobre los combustibles fósiles y es que, si bien es cierto que produce dióxido de carbono como los combustibles fósiles, no es un compuesto que aporte al calentamiento global debido a que es un compuesto biogénico [3]. La biomasa la podemos clasificar de la siguiente manera:

- Plantas leñosas: una planta leñosa es aquella que produce madera como tejido estructural y está formada principalmente por celulosa y lignina.

- Plantas herbáceas: este tipo de planta no tiene mucha madera, y sus ramas son verdes y suaves, crecen rápido y usualmente producen flores.

- Plantas acuáticas: estas plantas son aquellas que están adaptadas a vivir en ambientes acuáticos, ya sean de agua dulce o salada. Estas plantas solamente pueden crecer directamente en agua o en tierra que está permanentemente saturada con agua.

- Estiércoles: es derivado principalmente de las heces de animales, este material es rico en nutrientes como el nitrógeno, por lo cual funcionan muy bien como fertilizantes.

\subsection{Bagazo de caña de azúcar}

El bagazo de caña de azúcar es el residuo sólido remanente después de ser extraído el jugo de la caña de azúcar. Usualmente, es utilizado como combustible de los ingenios azucareros, pero debido a su alto contenido de celulosa también es aprovechado para la industria del papel. La estructura del bagazo de caña cuenta con dos partes: la fibra, que son el producto de la corteza y la parte interior del tallo una vez se ha procesado en un ingenio, y el meollo que es producto del tejido vegetal en el cual se encuentran los órganos vegetales. El bagazo de caña de azúcar, una vez ha 
sido procesado en el ingenio cuenta con la siguiente composición química [4]:

Tabla 1. Composición química del bagazo de caña

\begin{tabular}{|l|l|}
\hline Componente & Porcentaje \\
\hline Hidrógeno & $6.5 \%$ \\
\hline Oxígeno & $44 \%$ \\
\hline Carbono & $47 \%$ \\
\hline Cenizas & $2.5 \%$ \\
\hline
\end{tabular}

\subsection{Proceso de gasificación}

El proceso de gasificar residuos orgánicos para producir syngas es una tecnología de más de cien años de antigüedad. Este gas puede ser usado para generar potencia mediante turbinas, en equipos de calor y potencia (como en los ingenios azucareros), y hasta puede ser utilizado como combustible para ser quemado en motores de combustión interna.

El proceso de gasificación se lleva a cabo en cuatro partes, las cuales son el secado, pirólisis, oxidación y reducción.

\subsubsection{Secado}

En la fase de secado se remueve el agua en exceso que es contenida en la biomasa a una temperatura mayor a $100^{\circ} \mathrm{C}$. Esto ocurre debido a que en el momento en el que se introduce la biomasa al gasificador, recibe calor de los alrededores.

Esta parte del proceso es importante puesto que, aunque es posible gasificar biomasa con alto contenido de humedad, si se usa una cierta cantidad de energía para remover la humedad de esa materia, no habría manera de recuperar la energía, lo que haría al proceso menos eficiente. Es por esto que se aconseja dar un pretratamiento a la biomasa para evitar que tenga un nivel de humedad alto al introducirla al gasificador y que de esta manera se produzca un syngas con un poder calorífico mayor [2].

\subsubsection{Pirólisis}

Esta etapa consiste en la descomposición de materia orgánica mediante el calentamiento a altas temperaturas en ausencia de oxígeno. Este proceso se lleva a cabo a temperaturas entre 400 y $600 \mathrm{~K}$, por esto las grandes cadenas químicas de la biomasa se descomponen y dejan como residuo alquitrán, material volátil y carbón pirolisado, que tiene un mayor valor energético para la producción de syngas [6].

\subsubsection{Oxidación}

En esta etapa, se introduce un agente gasificante al sistema, usualmente se utiliza aire, oxígeno o vapor de agua para generar el syngas que consiste en su mayor parte por hidrógeno y monóxido de carbono. La relación entre oxígeno/carbono dentro del sistema influye mucho en la calidad del producto final, ya que mientras más oxígeno hay, más completa será la combustión [6].

\subsubsection{Reducción}

En esta parte del proceso se producen las reacciones químicas que generan el monóxido de carbono y el hidrógeno, que brindan un alto poder calorífico al syngas. El objetivo de este proceso es lograr las condiciones que favorecen a estas dos reacciones, ya que los compuestos resultantes son la mayoría de la composición del syngas [2].

Una vez se obtiene el syngas bruto del gasificador, éste tiene que pasar por un sistema de purificación para poder ser utilizado en la industria [5].

\subsection{Tipos de gasificadores}

Un gasificador es el equipo más importante en una planta de gasificación, debido a que es en este elemento donde se obtendrá el syngas que ha de ser usado posteriormente para la producción de energía, ya sea al quemarlo en una caldera, en un motor de combustión interna o almacenarlo en una celda de combustible. Este elemento en sí, es uno al que se le ha dedicado mucho tiempo en investigación, ya que, en conjunto con otros factores, tales como las propiedades de la biomasa y su pretratamiento y las condiciones de operación, afectarán directamente a la eficiencia del proceso de gasificación [3]. Los gasificadores pueden ser clasificados dentro de tres grupos: gasificadores de lecho fijo, de lecho fluido y de flujo arrastrado.

\subsubsection{Gasificadores de lecho fijo}

La característica principal de este tipo de gasificador es que la biomasa es suministrada a la cámara del reactor en grandes cantidades a través de una apertura, la cual se encuentra ubicada en la parte superior del reactor y desciende a través del mismo. A medida que ocurre la conversión del combustible en forma de gas, el residuo desciende por acción de la gravedad [3] [7]. Estos tipos de gasificadores son los más comunes, pero cuentan con la desventaja de que, debido a que trabajan a muy altas temperaturas (alrededor de $1000^{\circ} \mathrm{C}$ ), producen una mayor cantidad de alquitrán, lo que a su vez causa que el poder calorífico disminuya [8]. Los gasificadores de lecho fijo a su vez, se pueden clasificar dentro de tres grupos: gasificadores de flujo ascendente, de flujo descendente y de flujo cruzado y esta clasificación va a depender de la manera en la que se produzca el flujo del aire.

\subsubsection{Gasificador de flujo ascendente}

En estos tipos de gasificadores el reactor es alimentado desde la parte superior y el aire, oxígeno o vapor de agua entran por la parte inferior del gasificador; lo que significa que el gas se mueve en dirección contraria y por eso obtienen el nombre de gasificadores en contra corriente. El combustible pasa por una zona de "secado" en donde se elimina la humedad que este posee y se libera agua sin reaccionar. Seguido a esto, ocurre el proceso de pirólisis, en donde se da la descomposición del material en forma de gas y cenizas [3]. Este tipo de gasificador es frecuentemente utilizado en el caso de que el tipo de biomasa a utilizar presente un alto contenido 
de humedad, pero su desventaja es que produce un alto contenido de alquitrán [7]. La biomasa a utilizar en este tipo de gasificador debería contar con las siguientes características para que el proceso mantenga su eficiencia:

Tabla 2. Parámetros para la biomasa en gasificadores de flujo ascendente [3]

\begin{tabular}{|l|l|}
\hline \multicolumn{1}{|c|}{$\begin{array}{c}\text { Característica de la } \\
\text { biomasa }\end{array}$} & \multicolumn{1}{c|}{ Parámetro } \\
\hline Tamaño de la biomasa & $0.5-20 \mathrm{~cm}$ \\
\hline Contenido de agua & $<40 \%$ de la masa húmeda \\
\hline Contenido de cenizas & $<6 \%$ de la masa seca \\
\hline Tamaño de la fibra & $\leq 5 \mathrm{~mm}$ \\
\hline
\end{tabular}

\subsubsection{Gasificador de flujo descendente}

El gasificador de flujo descendente, es también denominado gasificador de corriente paralela. En este modelo el agente reactante (aire, vapor de agua u oxígeno) es suministrado por la parte media del gasificador, por lo cual el syngas es entregado por la parte inferior de este. Esto significa que el combustible y el gas se mueven en la misma dirección [1]. Su diferencia principal con el gasificador de flujo ascendente recae en que los gases liberados en el proceso de pirólisis intervienen en el proceso de gasificación, mientras que en los de flujo ascendente no ocurre esto. La principal desventaja de este modelo es el alto contenido de cenizas y humedad. Sin embargo, estos gasificadores cuentan con una ventaja y es que, si son operados a la temperatura apropiada, producen un gas que se puede utilizar en motores de combustión interna, ya que el gas generado tiene un bajo nivel de alquitrán. Esto se debe a que en el proceso de pirólisis se produce un gas que atraviesa una zona en la que la temperatura apropiada que mencionamos anteriormente $\left(800^{\circ} \mathrm{C}-1300^{\circ} \mathrm{C}\right)$, se encarga de craquear el alquitrán y de este modo se produce un mejor syngas [7] [8]. Al igual que en el gasificador de flujo ascendente, la biomasa a utilizar en este tipo de gasificador debería contar con las siguientes características para que el proceso mantenga su eficiencia:

Tabla 3. Parámetros para la biomasa en gasificadores de flujo descendente [3]

\begin{tabular}{|l|l|}
\hline \multicolumn{1}{|c|}{$\begin{array}{c}\text { Característica de la } \\
\text { biomasa }\end{array}$} & \multicolumn{1}{c|}{ Parámetro } \\
\hline Tamaño de la biomasa & $2-20 \mathrm{~cm}$ \\
\hline Contenido de agua & $\begin{array}{l}<10-25 \% \text { de la masa } \\
\text { húmeda }\end{array}$ \\
\hline Contenido de cenizas & $<6 \%$ de la masa seca \\
\hline Tamaño de la fibra & $\leq 5 \mathrm{~mm}$ \\
\hline
\end{tabular}

\subsubsection{Gasificador de flujo cruzado}

El gasificador de flujo cruzado es parecido al de flujo descendente, pero en este caso el agente reactante entra al gasificador mediante una garganta, la cual es ubicada en la parte inferior del éste y el gas producido entonces sale por el lado opuesto a la entrada del agente reactante [8]. Este tipo de gasificador tiene un tiempo de arranque corto, de aproximadamente cinco minutos, tiene la capacidad de operar con combustibles húmedos o secos, y la temperatura del producto final es alta. Por eso el contenido de hidrógeno y metano del syngas resultante es bajo [3].

\subsubsection{Gasificador de lecho fluido}

En este modelo, el agente gasificante es introducido por la parte inferior del sistema, y es también el gas de fluidización, y la biomasa es introducida por la parte superior del gasificador. La biomasa se encuentra en estado sólido y se mantiene en contacto constante con un catalizador, el cual puede encontrarse suspendido en un fluido, ya sea en estado líquido o en forma de gas. La biomasa es calentada casi instantáneamente a la temperatura que se requiere para las reacciones químicas, lo que causa que el proceso de pirólisis ocurra muy rápidamente y con una alta cantidad de gases [7]. Este tipo de reacción, que se mantiene en movimiento constante, asegura que el combustible se encuentre en contacto profundo con el catalizador. Una desventaja de este tipo de gasificador es el hecho de que, al ser una reacción homogénea, no permite dividir la reacción en diferentes áreas; sin embargo, posibilita el alcanzar altos niveles de potencia (100 MW).

\subsubsection{Gasificador de flujo arrastrado}

En este tipo de gasificador el proceso de gasificación es rápido ya que trabaja con temperaturas altas que oscilan entre 1200 y $1500^{\circ} \mathrm{C}$ [3]. Como resultado de este hecho, la pirólisis del combustible junto con la gasificación ocurren al mismo tiempo. Esto significa que esta tecnología trabaja con bajos niveles de oxígeno y por lo general suele funcionar con presiones que oscilan entre 20 a 70 bar [7].

\section{Materiales y métodos}

\subsection{Materiales}

Para realizar nuestra investigación necesitaremos una serie de equipos con los cuales realizaremos el pretratamiento a la biomasa para poder tener un bagazo de caña con las propiedades adecuadas para la producción de un syngas de alta calidad, dichos materiales son:

- Analizador de gas de síntesis, modelo Gasboard 30100P

- Picadora/triturador forrajero marca Trapp, modelo TRF-300.

- Pelletizadora de marca Torotrac con dado de orificios de $4 \mathrm{~mm}$ de diámetro

- Gasificador tipo flujo ascendente o descendente (en proceso de elección)

- Bagazo de caña de azúcar

- Termopares

- Soplete 


\subsection{Metodología}

Este proyecto de investigación consta de distintas fases: revisión bibliográfica, obtención de la biomasa de los distintos ingenios, pruebas con el gasificador y análisis del syngas producido, cuantificación de la energía producida por kilogramo de bagazo de caña de azúcar quemado en los distintos ingenios.

\subsubsection{Revisión bibliográfica}

En este punto de nuestro proyecto de investigación nos instruiremos en el tema de la producción de energía utilizando biomasa mediante el proceso de gasificación. Básicamente, leeremos publicaciones que se hayan hecho basadas en temas relacionados a nuestro enfoque, para poder extraer los conceptos necesarios de dichas publicaciones y artículos, de tal modo que podamos aplicarlos a nuestro proyecto.

\subsubsection{Obtención de la biomasa}

Para poder hacer un análisis competente del rendimiento energético del bagazo de caña, se tomó una muestra de por lo menos dos ingenios azucareros en Panamá, debido a que las propiedades de este tipo de biomasa varían dependiendo de muchos factores, entre ellos podemos mencionar el tipo de suelo en donde se planta la caña de azúcar, los fertilizantes y pesticidas que se usan para su cuidado. Es por esto, que el syngas que se obtendrá de uno u otro va a diferir, ya que las propiedades tales como su humedad, celulosa, carbono e hidrógeno pueden diferir por el tratamiento que se le dé en los sembradíos. Se obtuvieron quince sacos de bagazo de caña de la central azucarera de Alanje y diez sacos del ingenio Santa Rosa. Con esa cantidad de biomasa se realizaron distintas pruebas en los gasificadores.

\subsubsection{Pruebas de los gasificadores y análisis del syngas}

En esta etapa de la investigación se realizaron distintas pruebas en los gasificadores existentes en la sede de Tocumen de la Universidad Tecnológica de Panamá. Dichos gasificadores fueron modificados para mejorar su eficiencia, de tal modo que pudieran producir un mejor syngas. En estos dos gasificadores (flujo ascendente y descendente) no se realizaron pruebas con el bagazo de caña, sino que se usó virutas de madera para comprobar si eran capaces de producir syngas. Con estas pruebas se logró producir syngas, pero no en cantidades significativas. Por esta razón, se optó por adquirir un nuevo gasificador, pero se está decidiendo aún el tipo de gasificador que se importará. Sin embargo, de estas pruebas pudimos comprobar que se logró producir una pequeña cantidad de syngas con los gasificadores que aún están en proceso de restauración. Al momento de adquirir el nuevo gasificador, al darle un pretratamiento a la biomasa se conseguirá un mejor syngas. Además de esto, se adquirió equipo con el que se dará un pretratamiento al bagazo de caña para obtener una biomasa con mejores propiedades para utilizar en el gasificador, entre estos equipos podemos mencionar la picadora y la peletizadora.

\subsubsection{Cuantificación de la energía producida en los ingenios azucareros}

Para poder realizar una comparación entre el rendimiento energético que le brinda al ingenio quemar el bagazo de caña en una caldera, es necesario ir a los ingenios y obtener información de este proceso para poder saber que tanta energía producen. Una opción podría ser saber cuántos kilogramos de azúcar obtienen al quemar una cantidad de kilogramos de bagazo de caña de azúcar. Una vez se haya obtenido esta información, se procederá a hacer la comparación con la energía que puede producir nuestro syngas y así sugerir actualizaciones en los equipos de los ingenios para obtener un mejor rendimiento en sus procesos de planta.

\section{Resultados y discusión}

Los resultados que esperamos obtener una vez hayamos finalizado la etapa de experimentación son que el bagazo de caña de azúcar, al ser gasificado sea capaz de producir un syngas tan eficiente que produzca más energía que cuando se quema directamente en una caldera. De igual modo, esperamos que el pretratamiento del bagazo de caña de azúcar, al ser peletizado, sea más apto para mejorar la eficiencia del proceso de gasificación que cuando se gasifica el bagazo en su estado fibroso.

Otro resultado que deseamos obtener es poder conocer qué tipo de gasificador produce un mejor syngas y para esto es necesario seguir haciendo ciertas modificaciones en los gasificadores con los que contamos actualmente, hasta que llegue el nuevo equipo que se planea utilizar. Finalmente, al haber probado que la gasificación de este tipo de biomasa es un proceso que traería muchos beneficios a un ingenio azucarero, esperamos que nuestra sugerencia de actualizar la planta con un equipo que permita que sus procesos sean más eficientes sea aceptada, puesto que de esto depende que en un futuro su proceso de planta sea tan eficiente que haya un excedente de energía que podrían incluso utilizar para otros fines de la planta.

\section{Conclusiones}

La gasificación de biomasa es un proceso líder para la producción de un gas, que puede ser utilizado tanto para aplicaciones de calor centralizado, como para producción de energía eléctrica y recientemente, se ha destacado por presentar un nivel de competitividad alto contra los recursos no renovables. Esto se debe a que no solo la materia prima utilizada para el proceso presenta una gran disponibilidad a nivel mundial, en nuestro caso específico contamos con distintos ingenios azucareros alrededor de todo el país que siembran caña de azúcar en grandes cantidades, por lo que hacen que este tipo de materia, que además tiene un alto poder calorífico, sea una alternativa con un alto grado de aceptación para desplazar los recursos no renovables que son nocivos para el medio ambiente. 
Basados en todos los estudios que hemos visto de distintos autores que trabajan en la misma línea de investigación, pero con distintos tipos de biomasa, podemos ver que han obtenido resultados impactantes en este aspecto, debido a que han demostrado que es posible generar energía eléctrica con este tipo de tecnología en grandes cantidades. Prueba de ello es que actualmente, se cuenta con una planta de biomasa de más de $700 \mathrm{MW}$ en el Reino Unido.

Basados en los documentos revisados, podemos asegurar que este proyecto puede traer resultados positivos, no solo a los ingenios a quienes beneficiaría el hecho de poder producir más energía eléctrica para sus procesos de planta lo que implica que tendrían que comprar menos energía eléctrica para operar; sino que, esta puede ser la semilla que impulse el uso de este tipo de energía en Panamá, para dejar a un lado la dependencia que tenemos hacia los recursos no renovables que contribuyen al calentamiento global.

\section{AGRADECIMIENTOS}

A la Facultad de Ingeniería Mecánica de la Universidad Tecnológica de Panamá por facilitarnos sus instalaciones y educación para el desarrollo de este trabajo a través de la Jornada de Iniciación Científica (JIC). De igual forma, nuestra gratitud al Grupo de Investigaciones Energéticas y Ambientales (GIERA) por abrirnos sus puertas, por su soporte técnico y compartir con nosotros sus experiencias y oportunidades de investigación. Damos gracias a la Secretaria Nacional de Ciencias, Tecnología e Innovación (SENACYT) por el apoyo brindado mediante el financiamiento del proyecto de investigación $\mathrm{N}^{\circ}$ IDDS15-083, a cargo del Dr. Orlando Aguilar, de donde surgió esta iniciativa de investigación. Finalmente, agradecemos a nuestros padres por guiarnos y darnos la oportunidad de contribuir con el crecimiento y desarrollo de nuestro país.

\section{REFERENCIAS}

[1] McKendry, Peter, "Energy production from biomass (part 1): overview of biomass", Applied environmental research center, Colchester, UK, 2001.

[2] Castellanos, José U., Sierra, Fabio E., Guerrero, Carlos A., "Modelado del proceso de gasificación de biomasa para aprovechamiento energético: una revisión al estado de arte". Colombia, 2002.

[3] Friedrich Lettner, Helmut Timmerer, Peter Haselbacher, "biomass gasification - state of the art description", Intelligent energy-Europe, 2007.

[4] Martínez, Jorge, Díaz, Juan, "generación de combustible del bagazo de la caña de azúcar en panamá, mediante un proceso de gasificación: revisión teórico experimental. universidad tecnológica de panamá”, Panamá, 2010.

[5] Bocci, E., Sisinni, M., Moneti, M., Vecchione, L., Di Carlo, A., y Villarini, M. (2014). State of Art of Small-Scale Biomass Gasification Power Systems: A Review of the Different Typologies. Energy Procedia.

[6] Forero, Carlos A., Guerrero, Carlos A., Sierra, Fabio E., "Producción y uso de pellets de biomasa para la generación de energía térmica: una revisión a los modelos del proceso de gasificación", Colombia, 2012.

[7] McKendry, P. (2002c). Energy production from biomass (part 3): gasification technologies.

[8] García Fernández, Luis Eduardo. "Obtención de gas combustible a partir de la gasificación de biomasa en un reactor de lecho fijo" 2011. 